\title{
Le monde de la formation postgraduée et continue est en mouvement
}

Chaque année, le rapport de gestion est l'occasion de donner, à l'aide de faits et de chiffres, un aperçu du travail fourni par l'Institut suisse pour la formation médicale postgraduée et continue (ISFM). Il permet également de revenir sur les évolutions et les défis actuels en lien avec la formation postgraduée et continue. Les chiffres confirment que le volume de travail accompli par les équipes de l'institut ne cesse d'augmenter. Nous leur adressons nos plus chaleureux remerciements pour leur engagement. Elles s'emploient à traiter de manière aussi rapide qu'efficace les demandes d'octroi de titre, les reconnaissances d'établissements de formation postgraduée, les révisions des programmes de formation et bien plus encore, sans oublier les innombrables demandes de renseignements venant aussi bien de Suisse que de l'étranger, et l'organisation de pas moins de 150 visites d'établissements, élément primordial pour garantir la qualité.
La mise en application de la réglementation implique un nombre important de tâches administratives, mais le cœur d'activité de l'ISFM ne se résume en aucun cas à ce volet administratif. La rapidité avec laquelle le secteur de la santé évolue, les changements structurels que traversent les hôpitaux, le peu de temps et de ressources financières à disposition et les développements rapides au sein de nombreuses disciplines sont des enjeux qui imposent à la direction et au comité de l'ISFM d'anticiper et de réfléchir à des mesures pour actualiser le système de formation des médecins. A cela s'ajoutent deux projets ambitieux menés de front: le développement du logbook électronique pour tous les médecins en formation et l'extension de la plate-forme de formation continue à tous les médecins en exercice. Le présent rapport passe en revue l'éventail d'activités de l'ISFM.

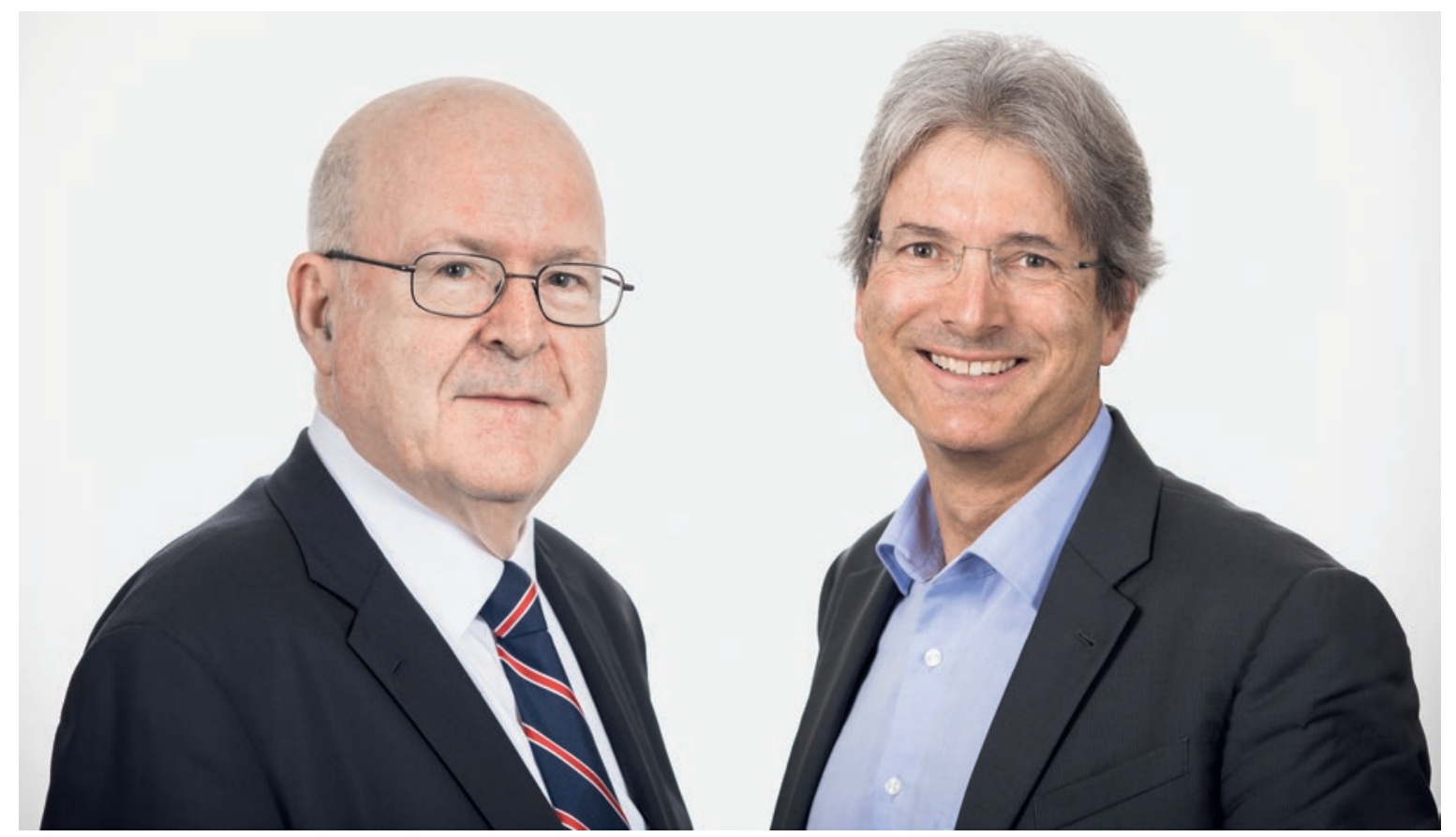

Le Dr méd. Werner Bauer, président de I'ISFM de 2010 à fin janvier 2021; Christoph Hänggeli, avocat, directeur de I'ISFM. 


\section{La formation médicale, le fonde- ment pour la qualité des soins de demain}

\begin{abstract}
La Chambre médicale a élu la Dre Monika Brodmann, p.-d. et MME, pour vous succéder. Quels sentiments associez-vous à ce passage de témoin?

Plusieurs. Tout d'abord, la chance d'avoir exercé la tâche la plus noble que le corps médical suisse puisse offrir à mes yeux. Ensuite, le plaisir de passer le flambeau à ma successeure car je sais que l'ISFM est entre de bonnes mains. Enfin, après un engagement de tous les instants pour l'ISFM, je dois me réorienter, sans préparation préalable alors que c'est ce que nous, médecins, n'arrêtons pas de recommander.
\end{abstract}

\section{Vous évoquez la tâche la plus noble du corps médical, pourquoi?}

Parce qu'il s'agit de la formation des médecins. Je suis persuadé que la bonne formation dispensée aujourd'hui aux futurs médecins spécialistes pose le fondement de la qualité des soins de demain. Cette qualité ne repose certes pas uniquement sur la compétence des médecins. De nombreux autres groupes professionnels doivent aussi apporter leur pierre à l'édifice mais dans

«La bonne formation dispensée aujourd'hui aux futurs médecins spécialistes pose le fondement de la qualité des soins de demain.»

les domaines de la prévention, du diagnostic, du traitement et du suivi, les médecins joueront toujours un rôle indispensable, que ce soit par leurs connaissances, leurs aptitudes ou leur engagement personnel; trois raisons pour lesquelles la formation postgraduée doit être reconnue, dispensée avec suffisamment de ressources et continuellement développée.

\section{Vous avez été le deuxième président de l'Institut suisse pour la formation médicale postgraduée et continue?}

C'est juste. L'ISFM a été fondé en 2009. Son premier président a été le Dr Max Giger, qui présidait déjà la Commission pour la formation postgraduée et continue, remplacée par l'ISFM, organe autonome de la FMH. Je suis entré en fonction en juin 2010 et ma mandature a été prolongée de six mois puisque les élections n'ont pas pu avoir lieu lors de la Chambre médicale du printemps 2020. J'ai donc vécu deux procédures d'accrédi- tation par laquelle le Département fédéral de l'intérieur certifie que l'ISFM et les sociétés de discipline médicale assument de bonne manière le mandat que l'Etat leur confie pour former les médecins spécialistes.

\section{Etiez-vous préparé à votre nouvelle mission et qu'avez-vous rencontré?}

Ce n'est pas sans un certain trac que j'ai commencé mon mandat, même si j'avais déjà une longue expérience dans le domaine de la formation médicale au sein d'organisations professionnelles helvétiques et européennes. J'ai très rapidement réalisé que notre système de formation couvrant plus de 100 titres de spécialiste, de formation approfondie et de formation complémentaire ne manquait pas de soulever des questions délicates concernant, pour la plupart, les reconnaissances que ce soit de titres, d'établissements de formation ou de stages accomplis à l'étranger, mais aussi la création ou les révisions de programmes de formation postgraduée. Mes débuts ont été facilités par l'appui d'une équipe très engagée et compétente, avec notamment le directeur, Christoph Hänggeli, et son adjointe, Barbara Linder.

\section{Quelles phases de la formation médicale sont du ressort de l'ISFM?}

La formation postgraduée, pour les spécialisations, et la formation continue, qui se rapproche de plus en plus du développement professionnel. Dans les pays anglosaxons, il est justement question de continuing professional development parce que les médecins de demain seront amenés à traverser des évolutions considérables entre l'obtention de leur titre de spécialiste et leur départ à la retraite. La formation continue vise donc à maintenir à niveau les compétences et à en acquérir de nouvelles. Aujourd'hui, on attend des médecins qu'ils en fournissent la preuve, ce qui peut être fait relativement facilement en ligne grâce au diplôme de formation continue de l'ISFM.

Inversement, l'ISFM n'est impliqué qu'indirectement dans la formation prégraduée, et ce lorsque les jeunes médecins fraîchement diplômés commencent leur assistanat et que nos responsables de la formation postgraduée leur confient leurs premières responsabilités. Nous ne devons pas perdre des yeux la continuité 


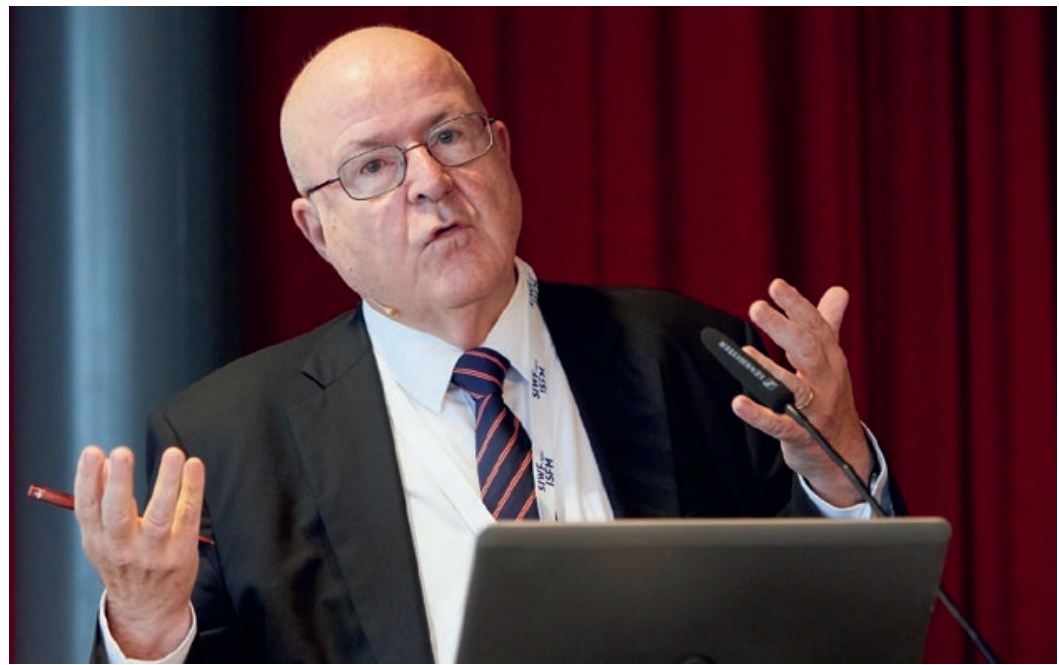

Werner Bauer a été président de I'ISFM de 2010 à fin janvier 2021.

entre ces trois phases (prégraduée, postgraduée et continue) et les concevoir aussi comme tel. C'est dans cet esprit que nous organisons tous les ans notre Journée de réflexion permettant aux représentants des facultés de médecine et de l'ISFM de se pencher conjointement avec les principales institutions partenaires (Office fédéral de la santé publique, Conférence des directrices et directeurs de la santé, Association des médecins-assistant(e)s et chef(fe)s de clinique, Académie suisse des sciences médicales, H+ Les hôpitaux de Suisse) sur un développement coordonné des études et de la formation postgraduée.

\section{Avez-vous pu innover?}

Innover participe certainement de la beauté d'une telle fonction. Je voulais des projets qui soutiennent la formation postgraduée de manière concrète mais aussi positionner l'ISFM pour qu'il devienne le centre suisse de compétence pour la formation postgraduée et continue. Nous avons ainsi créé un prix remis chaque année aux formatrices et formateurs engagés et lancé une promotion de projets pour laquelle près de 150000 francs sont attribués aux quatre à cinq projets sélectionnés tous les deux ans par le jury pour leur impact direct et positif sur la formation postgraduée. La plupart des dossiers sont d'une qualité admirable, si bien que le choix est rarement facile. Nous veillons cependant toujours à ce que le bénéfice ne soit pas que local.

FMH

Institut suisse pour la

formation médicale post-

graduée et continue (ISFM)

Nussbaumstrasse 29

Case postale

CH-3000 Berne 16

Tél. 0315030600

info[at]siwf.ch

\section{N'y a-t-il pas eu aussi les rencontres de l'ISFM?}

Tout à fait, le symposium MedEd et les ateliers de $\mathrm{Fa}$ culty Development. Une institution comme l'ISFM a besoin à mes yeux d'actions emblématiques, visibles de l'extérieur. C'est pourquoi nous avons lancé en 2014 le premier symposium MedEd sur les perspectives de la formation médicale. Entretemps, près de 200 personnes participent chaque année à cette rencontre et échangent sur les différents aspects de la formation médicale dans le cadre de conférences, de séminaires, de tables rondes, de débats et naturellement aussi pendant les pauses. Les retours ont toujours été jusqu'ici extrêmement positifs, ce qui est très réjouissant.

Les ateliers mis sur pied avec les maîtres de conférences du Royal College of Physicians of London à l'Hôpital cantonal de Lucerne sont aussi ce qu'on peut appeler une success story. Lorsque j'étais à la Fédération européenne de médecine interne, j'ai eu des contacts avec eux et comme une mission importante de notre institut consiste à former des formatrices et formateurs (teach the teachers), je me suis lancé en les invitant une première fois en 2012. Depuis, le succès de ces ateliers ne s'est plus démenti. Deux séries d'ateliers sont proposées chaque année sur des thèmes tels que Teaching in clinical settings, How to deal with an underperforming trainee ou Leadership for doctors. Même si ces cours sont en anglais, la demande est énorme et les échos très bons, notamment au sujet de leur applicabilité à la pratique helvétique. Nous sommes en train de mettre en place une équipe d'instructrices et d'instructeurs ici en Suisse pour compléter l'offre anglaise par des ateliers dans nos langues nationales.

\section{Que reste-t-il à faire pour votre successeure?}

Beaucoup de choses et pas des moindres. Le secteur de la santé, les institutions et les structures ambulatoires et hospitalières sont en pleine mutation, tout comme la formation. Elle devra donc veiller à ce que la formation soit adaptée aux conditions cadres sans concession sur la qualité; à ce que les objectifs de formation et les critères de reconnaissance suivent les évolutions de la médecine; à ce que la formation intègre de nouvelles méthodes telles que la simulation et la robotique et last but not least, poursuivre sur la voie didactique avec l'apprentissage axé sur les compétences comme le prônent les entrustable professional activities. C'est une belle mission et des tâches conséquentes que Monika Brodmann se réjouit certainement de relever. Je lui souhaite du fond du cœur plein succès et satisfaction.

\section{Crédits photos}

Tobias Schmid / ISFM (photo W. Bauer et Ch. Hänggeli) Heidi Fuchs, Medworld (photo W. Bauer)

\section{Version complète du rapport de gestion} de I'ISFM

Vous trouverez la version complète du rapport de gestion de I'ISFM incluant la rubrique "ISFM Compact» et une rétrospective du secrétariat de I'ISFM sur le site internet de I'ISFM: www.siwf.ch $\rightarrow$ L'ISFM en bref $\rightarrow$ Rapports de gestion 


\section{$\underset{\text { ISFM }}{\text { SIWEMan }} \mid \mathbf{2 0 2 0}$}

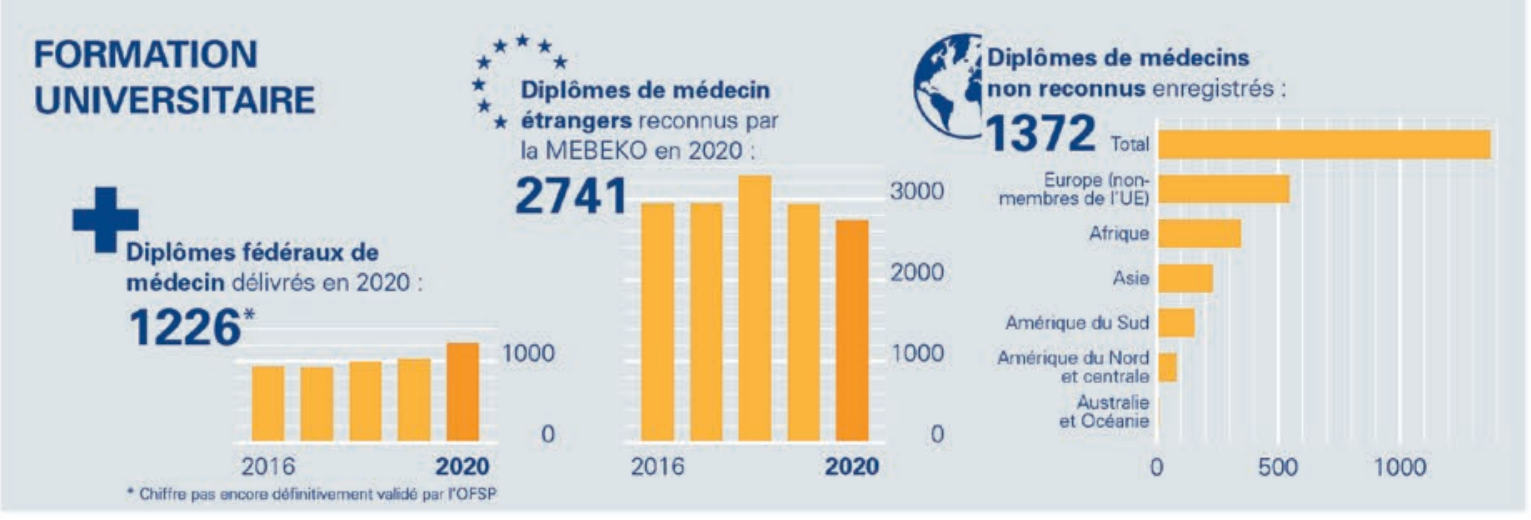

\section{FORMATION POSTGRADUÉE}

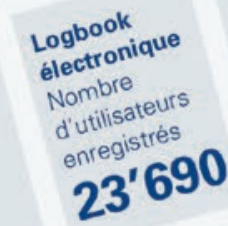

PROGRAMMES DE FORMATION POSTGRADUÉE

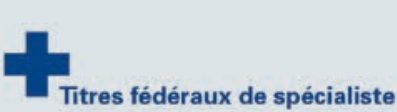
délivrés en 2020:

1687

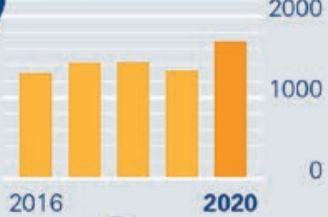

\section{Q 726 of 961}

\section{$\star^{\star \star \star} \star$}

Titres de formation

* postgraduée étrangers reconnus par la MEBEKO en 2020 :

\section{3} 2000

Les trois disciplines les plus fréquentes :

nombre de titres de spécialiste et

durée moyenne de formation

Médecine interne générale $586 / 6.7$ ans

Psychiatrie et psychothérapie

$131 / 8.1$ ans

Pédiatrie

$114 / 6,6$ ans

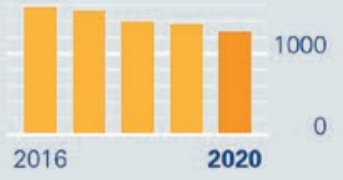

Formations approfondies

délivrées en 2020

239

Formations approfondies interdisciplinaires délivrées en 2020 :

98

\section{Attestations}

de formation complémentaire délivrées en 2020

Âge moyen des médecins qui obtiennent
leur premier titre de spécialiste, en tout : $\mathbf{3 6}, \mathbf{6}$ ans;

1208

\section{FORMATION CONTINUE}

\section{DIPLÔMES DE FORMATION CONTINUE}

saisis via la plate-forme
électronique de formation
continue et annoncés par continue et annoncés par
les sociétés de discipline :

\section{4}

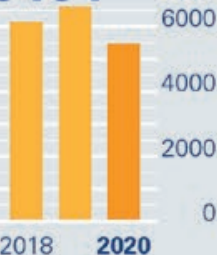

Médecins en exercice avec diplôme de formation continue valable

$43 \%$

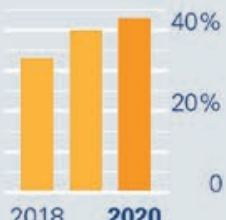

\section{SIWFFM \\ ISFM}

Nombre de sessions de formation continue reconnues par I'ISFM :

170

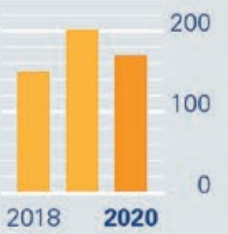




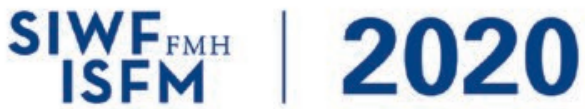

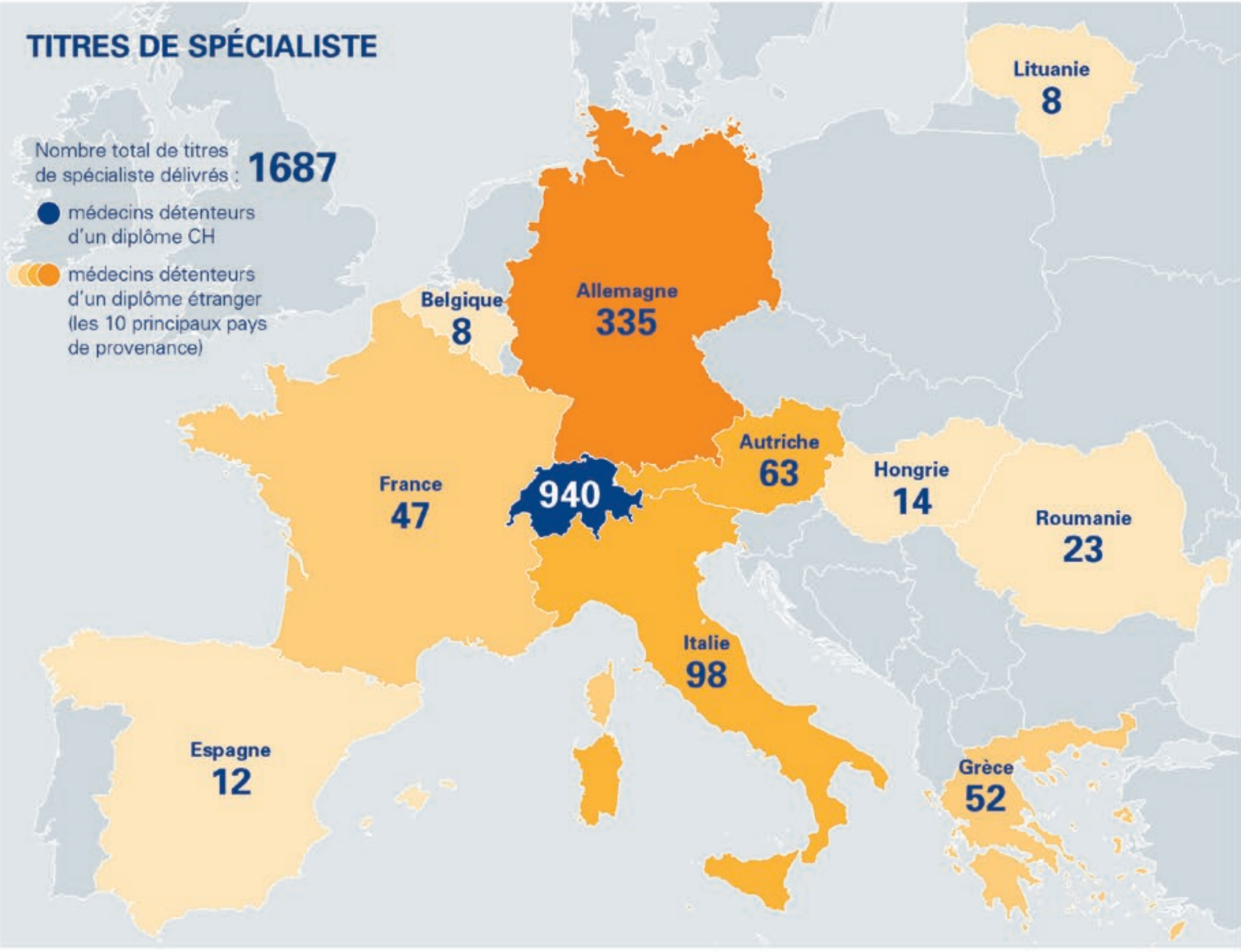

ENQUÊTE

AUPRÈS DES MÉDECINSASSISTANTS

\section{5}

médecins-assistants ont participé à l'enquête (taux de réponse $70.10 \%$ )

\section{4}

établissements ont été évalués

79 médecins sur 100 considèrent que les établissements de formation postgraduée contribuent beaucoup à énormément à la collaboration avec le personnel infirmier.

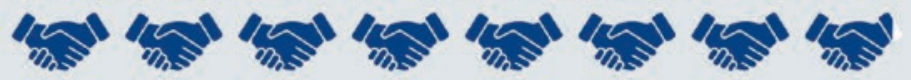

75 médecins sur 100 recommanderaient "entièrement " leur établissement de formation postgraduée, 19 sur 100 "plutôt ".
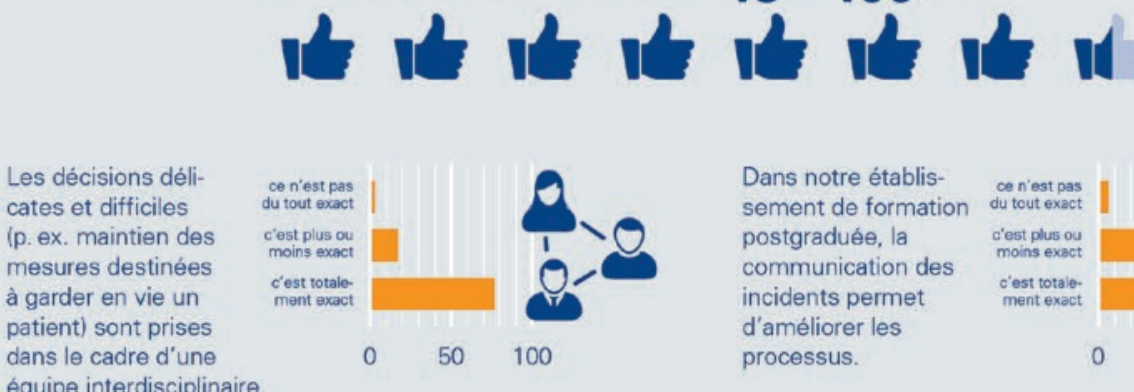
Dans notre établis- postgraduée, la communication des incidents permet d'améliorer les processus. cen'est pas

c'est plus ou

c'est totale

menteract

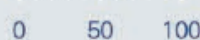

